\title{
Optimal Shape Design of the S-Shaped Subsonic Intake using NURBS
}

\author{
Byung Joon LEE ${ }^{1}$, Chongam $\mathrm{KIM}^{1}$ and Kyu-Hong $\mathrm{KIM}^{1}$ \\ 1) Department of Mechanical \& Aerospace Engineering, Seoul National University, Seoul \\ 151-742, KOREA
}

Corresponding Author: Chongam KIM, chongam@snu.ac.kr

\begin{abstract}
A parallelized design optimization approach is presented for a subsonic S-shaped intake using aerodynamic sensitivity analysis. Two-equation turbulence model is adopted to predict the strong counter vortices in the S-shaped duct more precisely. Sensitivity analysis is performed for the three-dimensional Navier-Stokes equations coupled with two-equation turbulence models using a discrete adjoint method. For code validation, the result of the flow solver is compared with experiment data and bench marking data of other computation researches. To study the influence of turbulence models and grid refinement in the duct flow analysis, the results using several turbulence models are compared with each other on various grid systems. The adjoint variable code is validated by comparison with the complex step derivative results. And to guarantee a sufficient design space, NURBS equations are applied as a new shape function to modify the duct geometry freely. The capability and the efficiency of the present design tools are successfully demonstrated in three-dimensional subsonic inlet flow analysis and design optimization.
\end{abstract}

\section{PRIMARY HEADING}

Both the commercial and the military aircrafts generally use the S-ducts to deliver the captured flow from far field to the engine compressor. The engine efficiency is influenced by the intake performance remarkably. Therefore, an efficient inlet duct should be able to deliver uniform and high pressure-recovery flow to the engine face. From the structural and aerodynamic point of view, it is more effective the length of inlet is short. The short length of duct, however, needs high curvature of the duct and highly curved duct generates large separated flows. To design an efficient intake, it is the most important problem to find the compromised optimum of the geometric constraints and the flow delivery performance of the intake. The performance of the intake is determined by: (1) incoming flow angularity with respect to the inlet cowl lip (2) uniform and high pressure recovery flow into the engine face (3) less vortex, wake and boundary layer ingestion by the inlet (4) interference with flows generated by other parts of the aircraft and ingested flow into the inlet etc. The engine face distortion represents the non-uniformity of the flow into the engine face and is one of the most important performance factors in the intake design. The non-uniform flow of the engine face is generated by the factors ingested from external flow field into the inlet, the secondary flow and flow separation within the duct itself.[1]

In this work, precise study of the phenomenon that captured flow is separated by the curvature of the duct and design optimization for an improved performance of the S-shaped diffuser are performed by flow analysis and sensitivity analysis using CFD. 
At first, about the flow analysis, the preceding researches are focused on precise prediction of the separation in the S-shaped duct by applying more refined grid systems and more suitable turbulence models. And they concluded that two-equation turbulence models predict the duct flow better than the algebraic and one-equation models do. In the present work, the flow analysis results from several two-equation turbulence models are compared. And grid refinement study is performed using various grid systems. Especially, for the last goal of this work, optimal shape design of the duct, both the efficiency and the accuracy of the flow solver are carefully investigated in the coarse grid system composed of 300,000 grid points.

Secondly, about the design optimization, there are many progresses about the shape optimization techniques of aerodynamic structure in the external transonic flow, i.e. airfoil and wing design. Recently, because of its excellent efficiency in case of a large number of design variables as like shape design, GBOM (Gradient Based Optimization Method) using adjoint variable method is in the spotlight for the shape design optimization. For a few reasons, however, this conventional design technique is not sufficiently implemented for the internal flow yet. As stated above, for accurate prediction of the large separated flow pattern in the duct, two-equation turbulence models should be applied to the flow analysis code and the sensitivity analysis. Due to the additional computational time cost for the two more turbulence equations, however, the sensitivity analysis of two-equation turbulence models have not been in the spotlight in the conventional external flow design. The difficulty in development of adjoint variable code is another cause for slow growth of duct shape design. Inevitably, in spite of its inefficiency, almost duct design works applied finite difference method which can be easily implemented. And this reason limits the design space to the local shape modification of the duct using Gaussian bump, vortex generator etc. These local modification techniques show a good performance to minimize the distortion but cannot make fundamental improvements in the duct flow as like total pressure recovery maximization. In this work, sensitivity analysis for two-equation turbulence model is performed using adjoint variable method to impose a large number of design variables and globally modify the duct geometry. And the inefficiency problem, which is caused by solving two more adjoint equations for turbulence equations, is settled by parallelized AV method. And the sensitivity analysis results are validated by the comparison with the complex step derivative results.

Lastly, new geometric representation functions are required in the surface modification. The conventional geometric representation methods based on the superposition, i.e. HicksHenne functions, Chebyshev polynomial, etc., are popularly used in the transonic wing section design. Because it conserves the smoothness of the airfoil line, although these methods are not compatible to secure a sufficient design space in case the geometric changes are severe. Jameson et al. used the grid points as design variables to increase the degree of freedom of geometric representation. However, grid points are inherently discontinuous, surface smoothness cannot be preserved. Therefore, they inevitably applied gradient smoothing, which may lessen the accuracy of the sensitivity analysis.

In case of the transonic wing design where the strong shock is observed on the wing upper surface, a small perturbation may enable the shock on the surface to vanish. On the other hand, in case of S-shaped duct design, the geometric variation is considerable to change the flow fundamentally in contrast with wing design case.

For the present design technique, therefore, NURBS (Non-Uniform Rational B-Spline) equation is applied as a new shape function. ${ }^{21}$ And the control points of NURBS surface and curve are used as design variables. NURBS can express any shape change of the duct surface. And the degree of freedom for the design space can be determined freely by the designer's requirements. Finally, NURBS can maintain the grid smoothness because NURBS equations can conserve higher order derivative continuity at every grid points. Hence, the gradient smoothing, which affects the accuracy of the gradients, is no more needed. 
Using these techniques, several cases of intake design problem are tested. The objective functions are the combinations of the total pressure recovery, the distortion, and the mass flow rate with weighting factors. In a subsonic intake, the flow change in the duct may affect the mass flow rate into the inlet from free-stream. To maintain flow condition, the mass flow rate in the form of a penalty function is combined to the objective function. The 'Distortion minimization with constant total pressure recovery' and the 'Total pressure recovery maximization' are performed to investigate the performance of the present automatic intake design technique.

\section{EQUATIONS, TABLES AND FIGURES}

Figure 1.

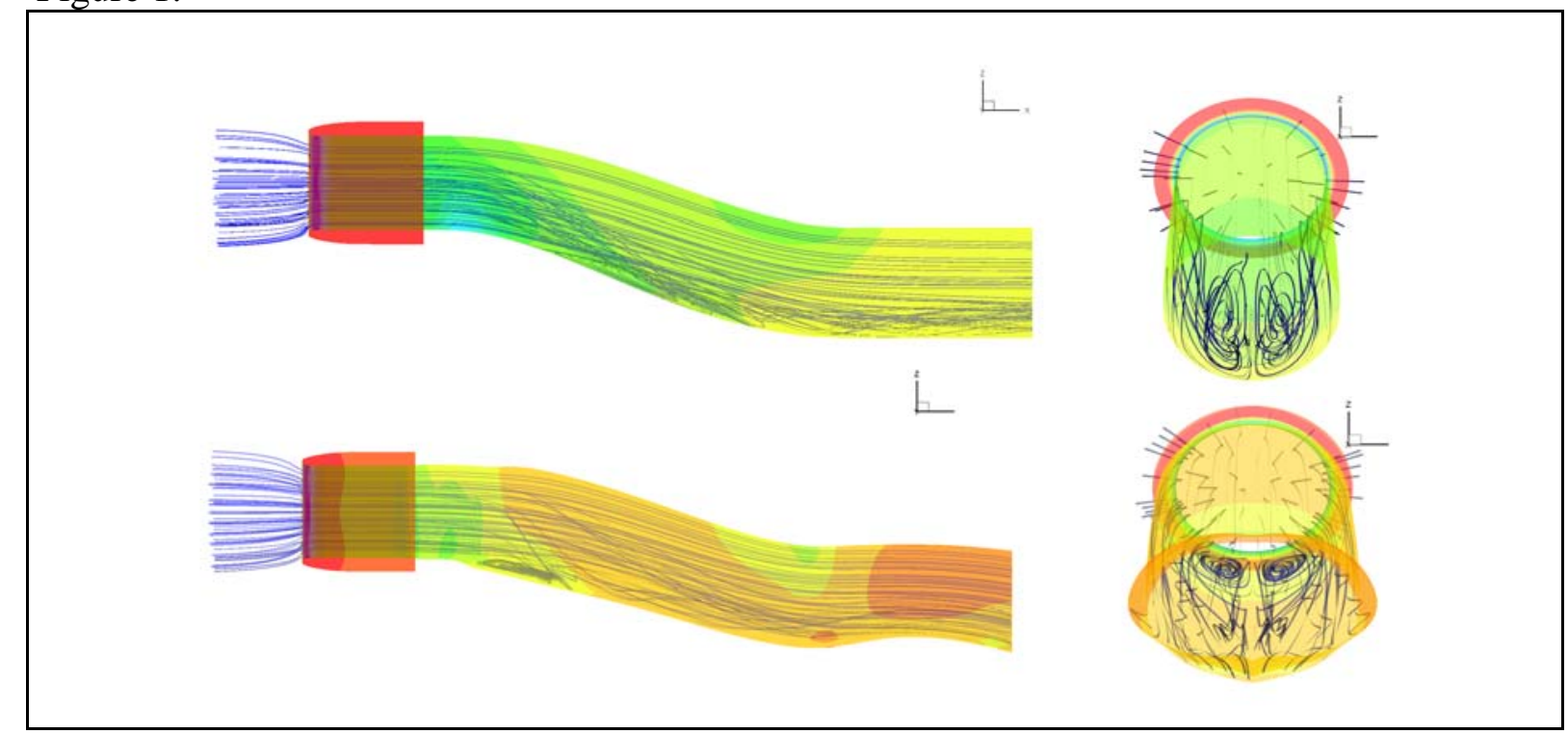

Figure 1. Shape \& Streamline Comparison (VATP Maximization, U:Baseline, L:Designed)

\section{REFERENCES}

(1) Book

1. J. Seddon, E. L. Goldsmith, “Intake Aerodynamics,” AIAA Education Series, 1985

2. Les Piegl , Wayne Tiller, "The NURBS Book,” Springer,1997.

(2) Paper in a journal

3. Zhang, W., Knight, D., and Smith, D., "Automated Design of a Three Dimensional Subsonic Diffuser," Journal of Propulsion and Power, Vol. 16, No. 6, 2000, pp. 11321140.

(3) Paper in Conference Proceedings

4. Fluid Dynamics Panel - Working Group 13. 1991 Test Case 3 - Subsonic/Transonic Circular Intake. AGARD Advisory Report 270 